\title{
Compensatory treatment of Angle Class III malocclusion with anterior open bite and mandibular asymmetry
}

Marcio Costa Sobral ${ }^{1}$

Class III malocclusion is characterized by anterior posterior dental disharmony, either with or without skeletal discrepancies. Facial esthetics may be compromised to a greater or lesser degree, depending on the magnitude of the discrepancy, and is one of the main factors motivating individuals to seek orthodontic treatment. In adult patients, therapy may be performed by means of dental compensation, in simpler cases, or in more severe situations, by means of association between Orthodontics and Orthognathic Surgery. The present article is a clinical case report of a patient with a vertical facial pattern, Angle Class III malocclusion, with open bite and important facial asymmetry. The patient was treated in a compensatory manner with extractions, using extra-oral appliances on the mandibular arch with high pull, applying the principles of the Tweed-Merrifield technique. This case was presented to the Brazilian Board of Orthodontics and Facial Orthopedics (BBO) as part of the requisites for becoming a BBO Diplomate.

Keywords: Facial asymmetry. Orthodontics. Angle Class III malocclusion.

${ }^{1}$ MSc in Orthodontics, Federal University of Rio de Janeiro. Professor of the Specialization Course in Orthodontics, Federal University of Bahia.
How to cite this article: Sobral MC. Compensatory treatment of Angle Class III malocclusion with anterior open bite and mandibular asymmetry. Dental Press J Orthod. 2012 May-June;17(3):138-45. 


\section{HISTORY AND ETIOLOGY}

The patient presented for initial exam at the age of 20 years, in a good state of general health. She had no abnormal pressure habit and the main complaint was related to the presence of open bite in the anterior region and facial asymmetry with mandibular deviation to the right side. The patient appeared to be concerned about facial esthetics, by virtue of the asymmetry caused by laterognathism (Fig 1). On a more detailed examination of the occlusion, true mandibular deviation to the left was found, probably generated by asymmetrical growth and not by a purely functional deviation. Although the mother did not report any family history of Class III, the peculiarities involved pointed towards a multifactorial etiology.

\section{DIAGNOSIS}

Regarding to facial characteristics, the patient presented a dolichocephalic facial type, with convex profile, slightly increased lower facial third, labial competence and presence of asymmetry due to mandibular deviation to the left side. The lips were protruded, and the bottom lip was slightly forward to the upper lip (Fig 1).

With regard to the dental aspect, the patient presented Angle Class III malocclusion, anterior open bite, overjet of $1 \mathrm{~mm}$ with projected mandibular and maxillary incisors, characterizing dentoalveolar double protrusion. In addition the maxillary arch was found to be atresic with slight anterior crowding and rotation of teeth 15 and 25 . The lower midline had a $2.5 \mathrm{~mm}$ deviation to the left side, but was coincident
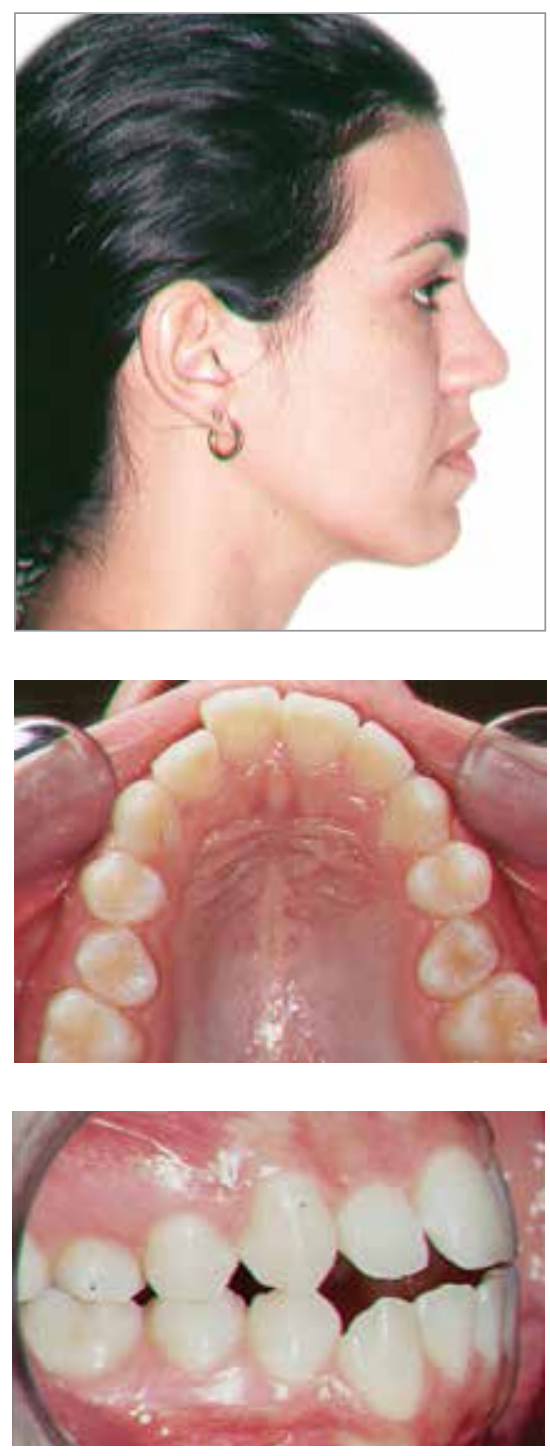
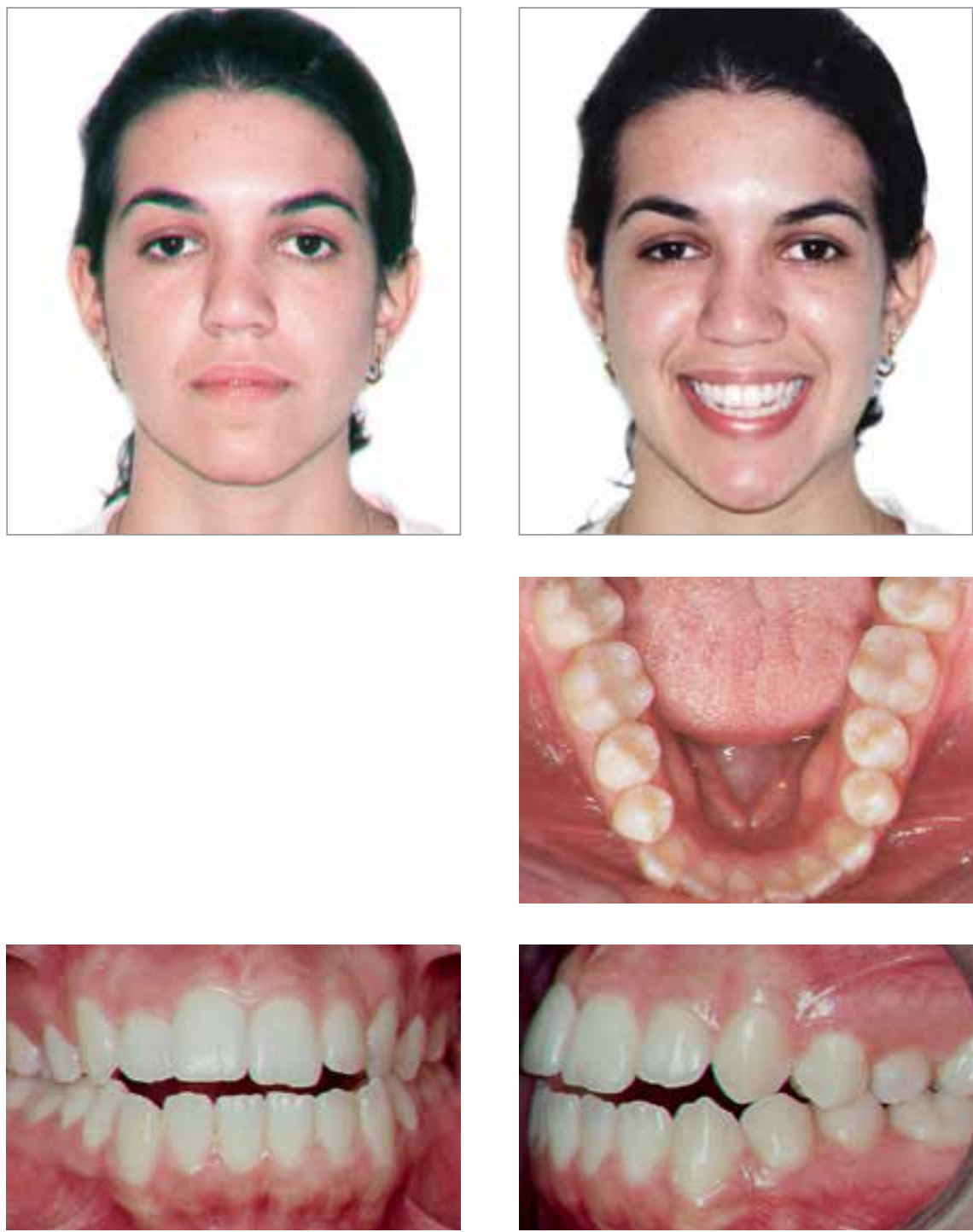

Figure 1 - Initial facial and intraoral photographs. 
with the center of the chin, characterizing a skeletal and not a dental deviation (Figs 1, 2).

The analysis of panoramic radiograph showed no significant alteration that would contraindicate performing orthodontic treatment (Fig 3). Cephalometric evaluation indicated important skeletal disharmony, with $\mathrm{ANB}$ equal to $-2^{\circ}\left(\mathrm{SNA}=78^{\circ}\right.$ and $\left.\mathrm{SNB}=80^{\circ}\right)$, poor mandibular growth in the vertical direction $\left(\mathrm{SN}-\mathrm{GoGn}=39^{\circ}\right)$ denoting the vertical aspect of the face. Maxilla and mandible were shown to be slightly retracted in relation to the cranial base. These observations may be better evaluated in Fig 4 and Table 1.

\section{TREATMENT OBJECTIVES}

In the maxilla, to promote transverse expansion with the aim of improving arch shape. Perform extraction of teeth 15 and 25, with the objective of simultaneously provide anchorage loss and incisors retraction, thus establishing key relation of occlusion between first molars and closure of open bite in the anterior region, respectively.

In the mandible, to promote efficient anchorage and vertical control as tooth 43 is moved in the distal direction, the midline is corrected and the incisors are retracted after extraction of teeth 34 and 44 . Thereby, significant improvement in the dental pattern was
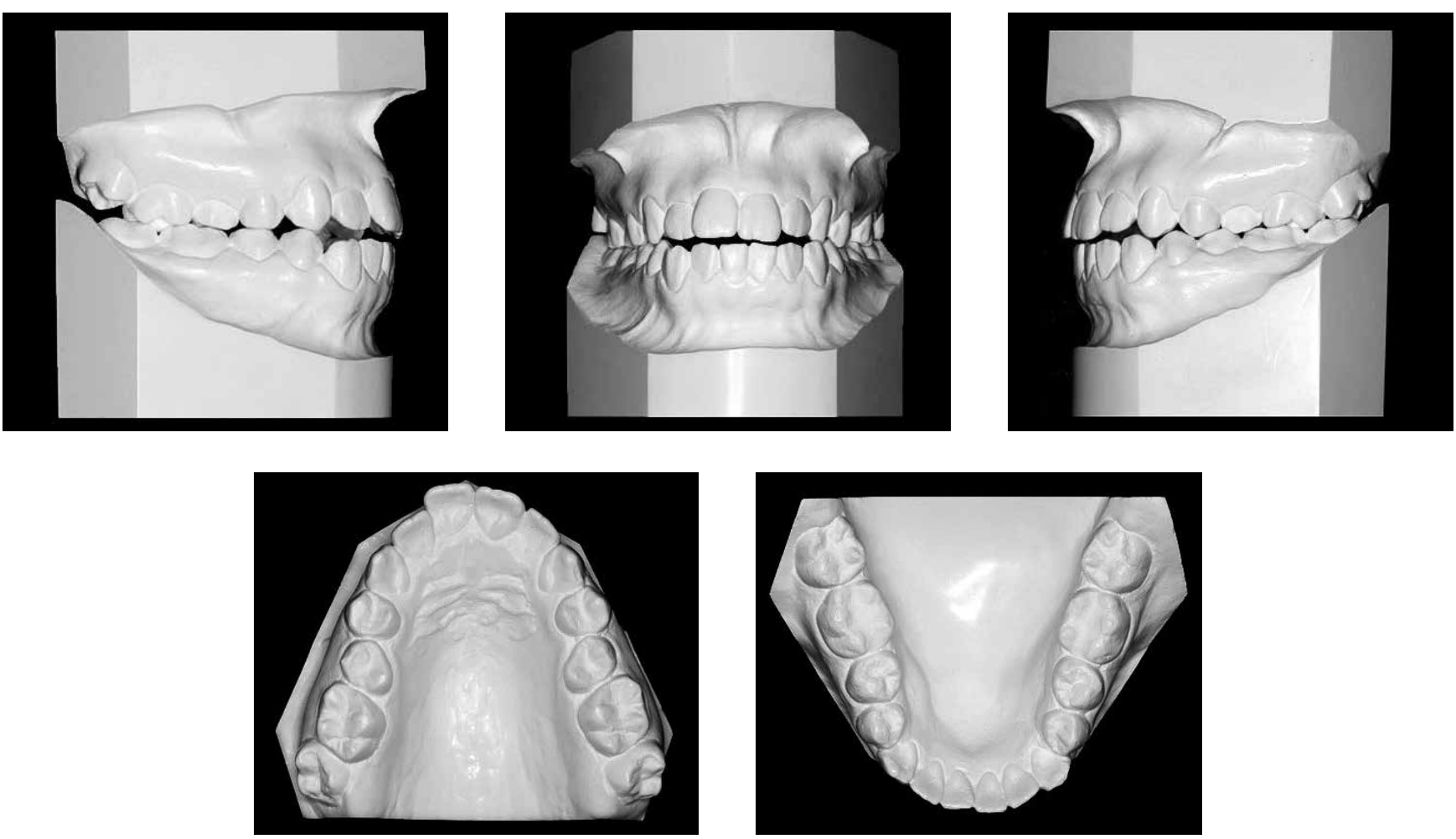

Figure 2 - Initial casts

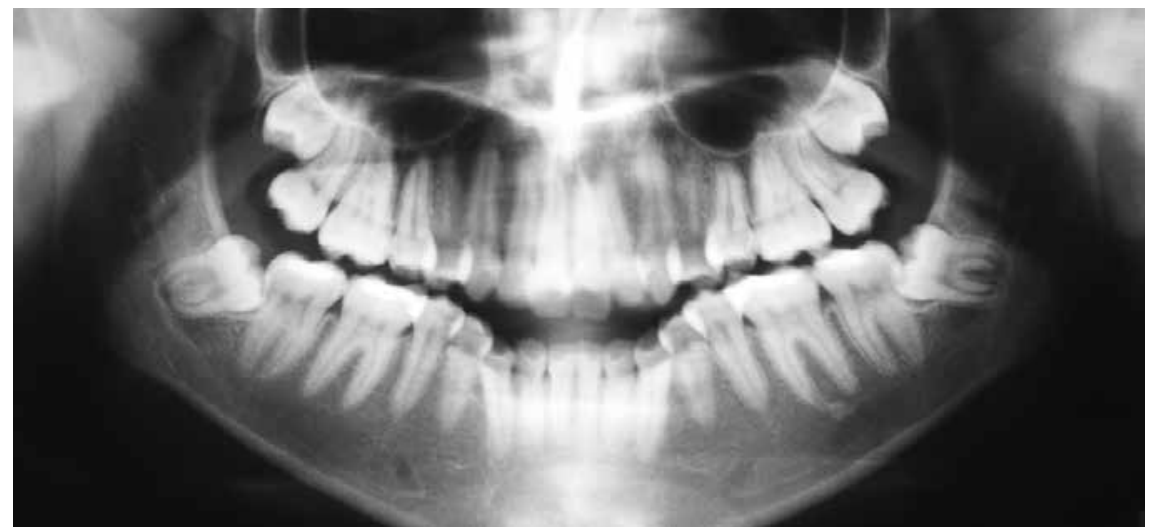

Figure 3 - Initial panoramic radiographs. 

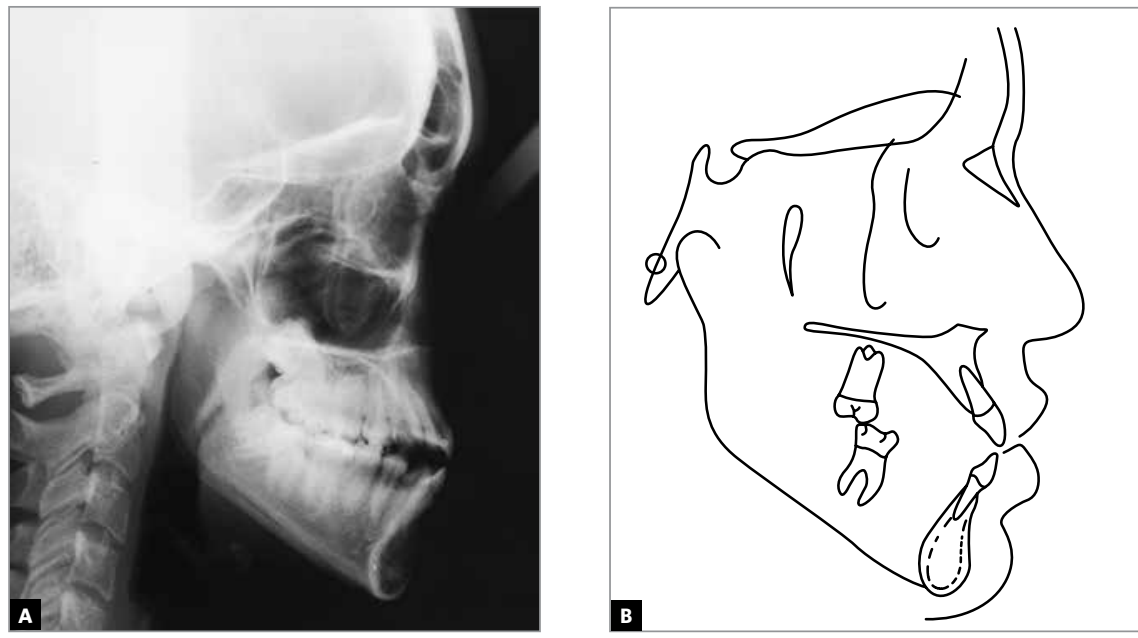

Figure 4 - (A) Initial lateral cephalometric radiograph and (B) cephalometric tracing.

expected with direct repercussion on the smile, however, without great alterations in the relationship of skeletal asymmetry between the mandible and maxilla.

\section{TREATMENT PLAN}

Two treatment plans were prepared. The first consisted of combined orthodontic-surgical treatment. The patient and his guardians expressed strong rejection of the surgical alternative and asked for another possibility. The other option would be an orthodontic camouflage, with extraction of four permanent teeth and the use of extra-oral appliances. In view of this, the guardians opted for the attempt to perform compensatory treatment and completely discarded the surgical approach.

In the beginning, slow expansion of the maxillary arch with the Hyrax expander appliance was planned. After this, a fixed total appliance with the standard Edgewise system would be placed, requiring extraction of teeth 15, 25, 34 and 44. After the initial stage of alignment and leveling, with the $0.018 \times 0.025$-in stainless steel rectangular arches already in place, a J-hook extra-oral appliance would be adapted to the mandibular arch, with high pull direction. This appliance would be anchored directly on the arch, touching the canines, functioning as jigs, with the objective of distalizing the mandibular canines and, simultaneously, due to the high pull, promote efficient vertical control favoring rotation of the mandibular occlusal plane in the counterclockwise direction, which would be favorable to the closure of the open bite. Due to the asymmetry and greater need for movement of tooth 43 in the distal direction, after distalization of tooth 33 , the J-hook would be anchored to a hook welded on the arch between teeth 32 and 33, while the right side would continue to play the role of a jig in moving tooth 43 .

Meanwhile, in the maxillary arch, closure of spaces would be conducted in a reciprocal manner, with the object of enabling the loss of posterior anchorage in conjunction with retraction and disinclination of the incisors and consequent closure of the open bite.

After this, $0.019 \times 0.026$-in stainless steel maxillary and mandibular arches would be made, with individualized bends and torques as required and, if indicated, the use of intermaxillary elastics for finishing. Retention in the maxillary and mandibular arches would be performed with wraparound removable retainers.

\section{PROGRESS OF TREATMENT}

In the maxillary arch, a modified Hyrax expander appliance was used with bands on the first molars and an extension bonded to the first premolars. After this, Edgewise standard metal brackets, slot $0.022 \times 0.028$ in, were bonded without torques or angulations. In the mandibular arch, in addition to the fixed appliance, the J-hook extraoral appliance with high pull was used.

Expansion occurred by means of activation by $1 / 4$ turns on alternate days for a period of 30 days. After the active period, the screw was stabilized and the appliance kept in place for three months. After removal of the Hyrax appliance, extraction of teeth 15 and 25 was required and alignment and leveling was performed with a sequence of 0.014-in, 0.016-in, 0.018-in and 
0.020-in stainless steel arches. In the mandibular arch, extraction of teeth 34 and 44 was initially required and alignment and leveling was started with a sequence of 0.014-in, 0.016-in, 0.018-in and 0.020-in stainless steel arches. When $0.018 \times 0.025$-in rectangular archwires were placed, the J-hook was adapted to the mandibular arch with high pull direction (150 g/side). The patient was instructed to use it for a minimum period of 12 hours/day. This appliance was anchored directly on the arch, touching the canines, working as jigs with the purpose of distalizing the mandibular canines and simultaneously to favor rotation of the mandibular occlusal plane in the counterclockwise direction, which would be favorable to closing the open bite. Due to the asymmetry and the need for greater distalization of tooth 33 , after distalization of tooth 33 , the $\mathrm{J}$ hook was anchored to a hook welded to the arch between teeth 32 and 33, while the right side continued to play the role of a jig distalizing tooth 43 .

Concomitantly, in the maxillary arch, closure of the spaces was performed in a reciprocal manner, with the objective of obtaining posterior anchorage loss together with retraction and uprighting of the incisors, and consequent open bite closure. After this $0.019 \times 0.026$-in
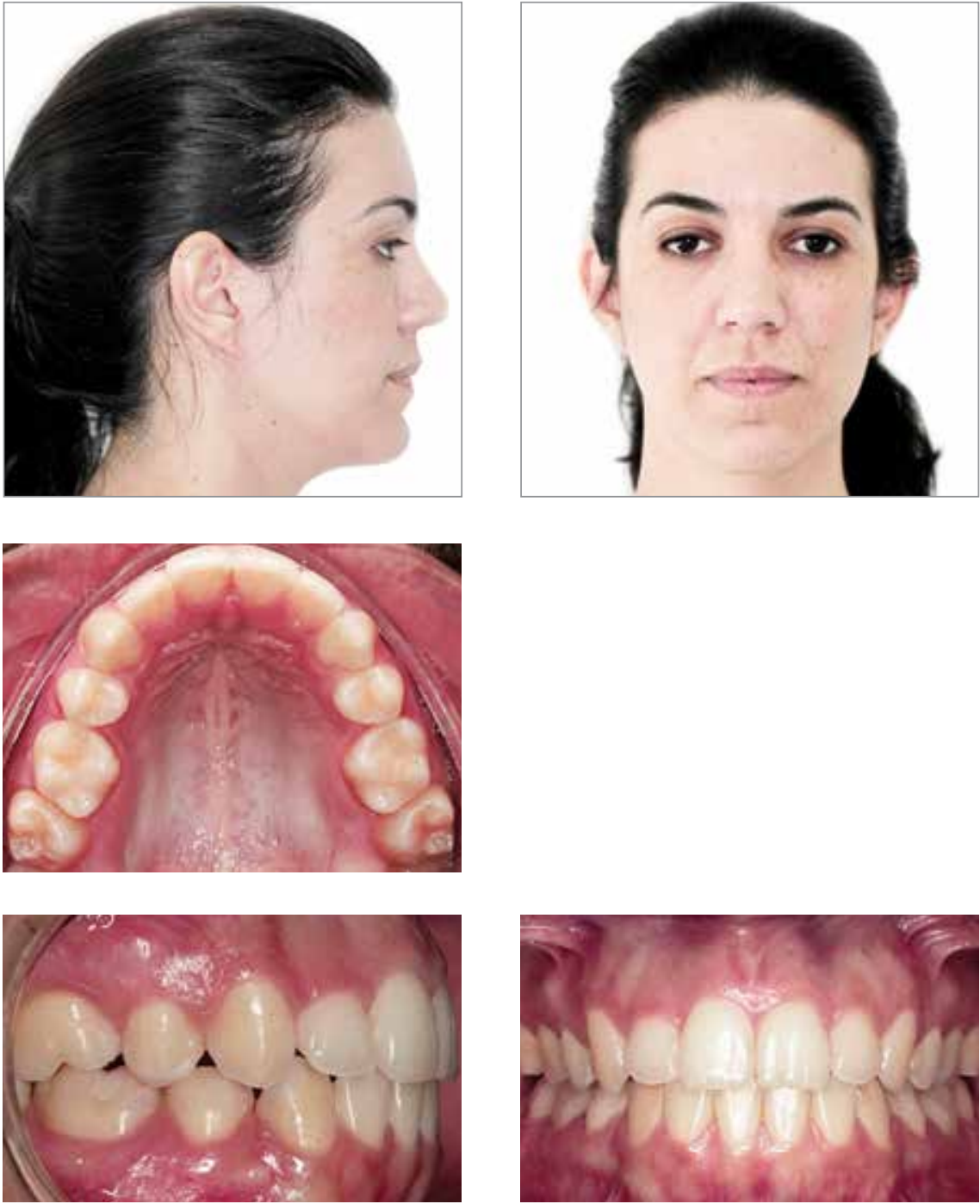
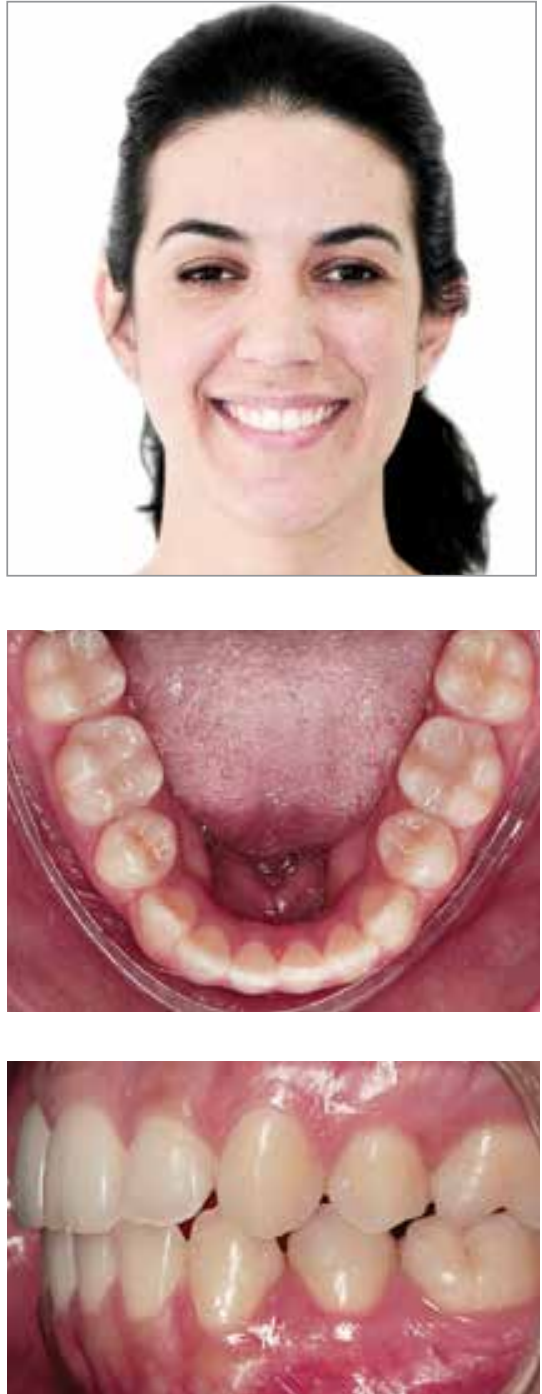

Figure 5 - Final facial and intraoral photographs. 
stainless steel mandibular and maxillary arches with individualized bends and torques were made, as required for finishing. Retention in the maxillary and mandibular arches was performed with wraparound type removable retainers.

\section{TREATMENT EVALUATION}

The main treatment objectives were attained, establishing an adequate dental relationship with important repercussion on general facial esthetics and in a more specific manner, significant improvement in the esthetics of the smile, with absence of exposure of the mandibular teeth (Fig 5), helping to camouflage mandibular asymmetry. It is worth pointing out that a preponderant factor for this successful treatment was the patient's cooperation with the use of extraoral mechanics. With the dental alterations, there was significant change in ANB angle from $-2^{\circ}$ to $3^{\circ}$ (Figs 8, 9 and Tab. 1). This fact can be attributed to remodeling of the alveolar processes in the maxillary and mandibular anterior regions in response to the retraction mechanics used. There was also significant improvement in the inclination of the mandibular and maxillary incisors, with reduction in $\underline{1}-\mathrm{NA}$ angle from $34^{\circ}$ to $18^{\circ}$
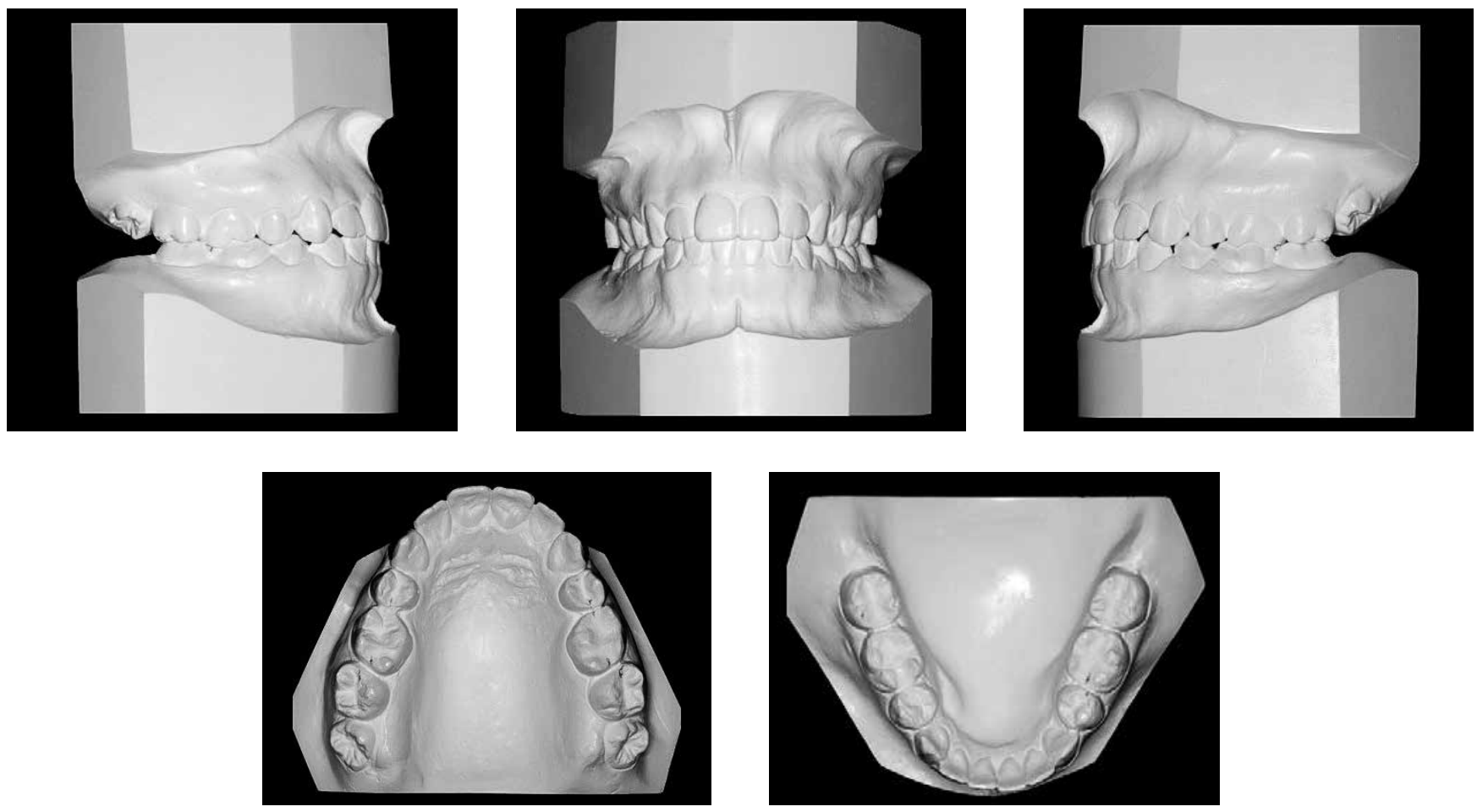

Figure 6 - Final casts.

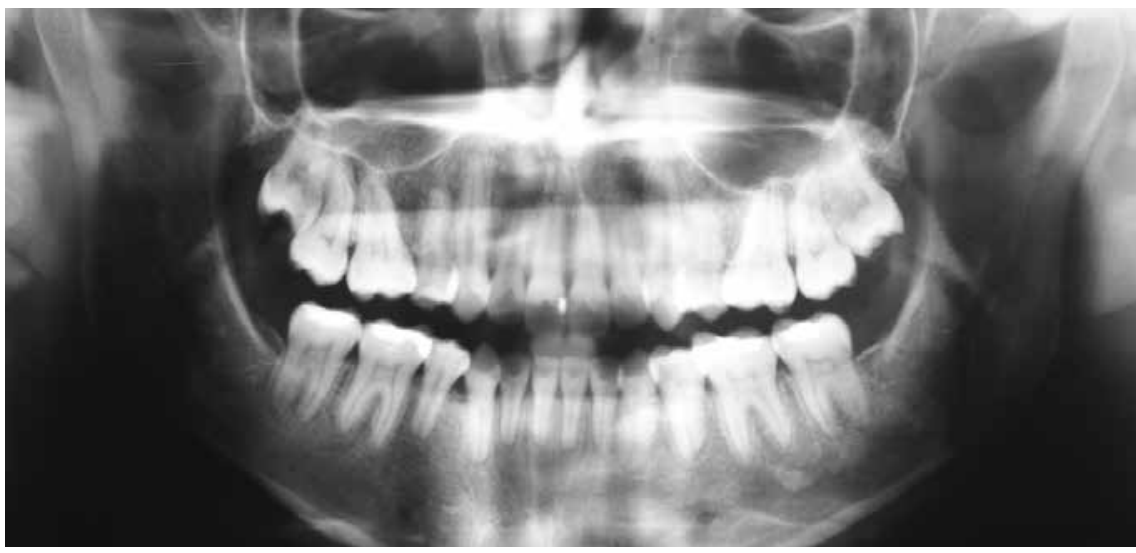

Figure 7 - Final facial and intraoral photographs. 

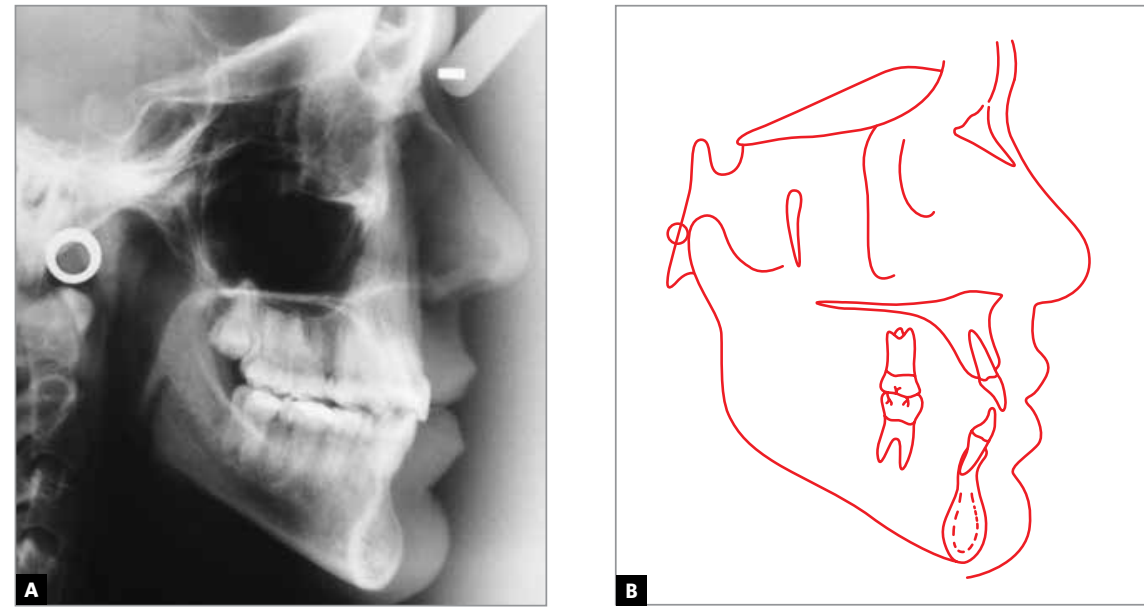

Figure 8 - A) Final lateral cephalometric radiograph and B) cephalometric tracing.
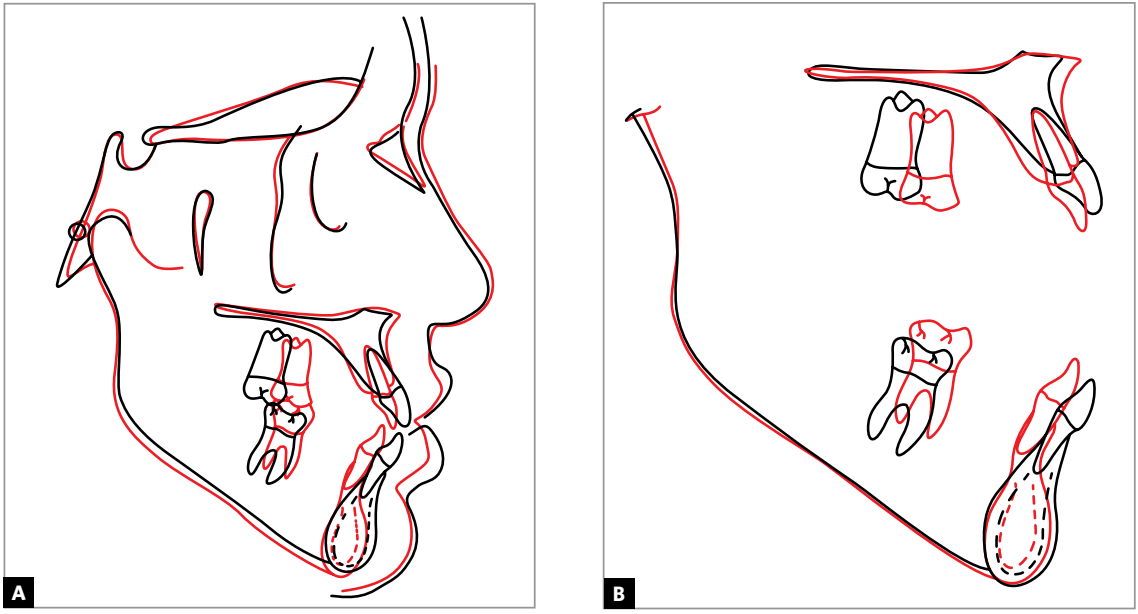

Figure 9 - A) Complete superimposition of the initial (black) and final (red) cephalometric tracings. B) Partial superimpositions: Maxilla and mandible.

Table 1 - Summary of cephalometric measurements.

\begin{tabular}{|c|c|c|c|c|c|c|}
\hline & \multicolumn{2}{|c|}{ Measures } & Normal & $\mathbf{A}$ & B & A/B Difference \\
\hline \multirow{8}{*}{ Skeletal pattern } & SNA & (Steiner) & $82^{\circ}$ & $78^{\circ}$ & $79^{\circ}$ & 1 \\
\hline & SNB & (Steiner) & $80^{\circ}$ & $80^{\circ}$ & $76^{\circ}$ & 4 \\
\hline & ANB & (Steiner) & $2^{\circ}$ & $-2^{\circ}$ & $3^{\circ}$ & 5 \\
\hline & Convexity angle & (Downs) & $0^{\circ}$ & $-3^{\circ}$ & $2^{\circ}$ & 5 \\
\hline & $\mathrm{Y}$ axis angle $\mathrm{Y}$ & (Downs) & $59^{\circ}$ & $66^{\circ}$ & $69^{\circ}$ & 3 \\
\hline & Facial angle & (Downs) & $87^{\circ}$ & $82^{\circ}$ & $80^{\circ}$ & 2 \\
\hline & Sn-GoGn & (Steiner) & $32^{\circ}$ & $39^{\circ}$ & $40^{\circ}$ & 1 \\
\hline & FMA & (Tweed) & $25^{\circ}$ & $37^{\circ}$ & $40^{\circ}$ & 3 \\
\hline \multirow{7}{*}{ Dental pattern } & IMPA & (Tweed) & $90^{\circ}$ & $93^{\circ}$ & $86^{\circ}$ & 7 \\
\hline & 1-NA (degrees) & (Steiner) & $22^{\circ}$ & $30^{\circ}$ & $20^{\circ}$ & 10 \\
\hline & $1-\mathrm{NA}(\mathrm{mm})$ & (Steiner) & $4 \mathrm{~mm}$ & $9 \mathrm{~mm}$ & $5 \mathrm{~mm}$ & 4 \\
\hline & T-NB (degrees) & (Steiner) & $25^{\circ}$ & $32^{\circ}$ & $24^{\circ}$ & 8 \\
\hline & T-NB (mm) & (Steiner) & $4 \mathrm{~mm}$ & $7 \mathrm{~mm}$ & $6 \mathrm{~mm}$ & 1 \\
\hline & $\frac{1}{1}$ - Interincisal angle & (Downs) & $130^{\circ}$ & $116^{\circ}$ & $137^{\circ}$ & 21 \\
\hline & T-APo $(\mathrm{mm})$ & (Ricketts) & $1 \mathrm{~mm}$ & $7 \mathrm{~mm}$ & $2 \mathrm{~mm}$ & 5 \\
\hline \multirow{2}{*}{ Profile } & Upper lip - S line & (Steiner) & $0 \mathrm{~mm}$ & $1 \mathrm{~mm}$ & $-1 \mathrm{~mm}$ & 2 \\
\hline & Lower lip - S line & (Steiner) & $0 \mathrm{~mm}$ & $3 \mathrm{~mm}$ & $-0,5 \mathrm{~mm}$ & 3,5 \\
\hline
\end{tabular}


and of $1-\mathrm{NB}$ angle from $32^{\circ}$ to $24^{\circ}$, with direct repercussion on the closure of open bite and improvement in facial profile (Fig 9 and Table 1). Correct occlusal relationships were obtained for canines and molars and the anterior open bite was corrected. Alignment, leveling and correction of rotations and inclinations were successfully achieved (Figs 5, 6).

\section{FINAL CONSIDERATIONS}

The presence of Angle Class III malocclusion, associated with the skeletal discrepancy is a delicate problem in the sphere of Orthodontics. ${ }^{1}$ Depending on the magnitude of this discrepancy and the degree of problem of facial esthetics, this problem could have negative psychological repercussions on the social life of an individual, in addition to the functional implications directly related to the stomatognathic system. ${ }^{3,4,5}$

There are a series of therapeutic resources in Orthodontics for the treatment of Class III malocclusion, which range from interception, for individuals at a early age, all the way to orthodontic-surgical treatment in adults. As an alternative, compensatory orthodontic treatment, also known as orthodontic camouflage, may be applied in certain cases. The main objective of this is to favor satisfactory occlusion by means of dental compensations, however with hardly significant changes in facial esthetics.
In the case described, the patient aging 20 years presented important facial asymmetry and in spite of being informed about the benefit of combined orthodontic-surgical treatment, she and her parents opted for orthodontic camouflage, although they were aware of the limitations of this procedure alone. The nonexistence of family history of similar discrepancy, as well as the report of the patient of being completely prepared to use the orthodontic mechanics provided by the extraoral appliances, were determinant factors in making the decision regarding therapy.

The treatment was performed with tooth extractions, expansion of the maxillary arch and extraoral mechanics acting directly on the mandibular arch, with the purpose of moving the mandibular teeth in the distal direction, correcting the Class III malocclusion and establishing adequate intercuspidation, without side effects on the maxillary arch, thereby applying a modification of the technique described by Tweed-Merrifield. ${ }^{2}$ With expansion of the maxillary arch and reciprocal space closure mechanics, after the extraction of teeth 15 and 25, it was possible to establish correct occlusal relationship for the first molars and canines, as well as adequate levels of overbite and overjet. With the correction of anterior open bite, there was significant improvement in the esthetic of the smile (Fig 5).

\section{REFERENCES}

1. Ellis E 3rd, McNamara JA Jr. Components of adult Class III malocclusion. J Oral Maxillofac Surg. 1984 May;42(5):295-305.

2. Merrifield LL. Edgewise sequential directional force technology. J Charles H. Tweed Int Found. 1986 Apr;14:22-37.

3. Ngan P, Wei SH, Hagg U, Yiu CK, Merwin D, Stickel B. Effect of protraction headgear on Class III malocclusion. Quintessence Int. 1992 Mar;23(3):197-207.

4. Turley PK. Orthopedic correction of Class III malocclusion with palatal expansion and custom protraction headgear. J Clin Orthod. 1988 May;22(5):314-25.

5. Tollaro I, Baccetti T, Franchi L. Mandibular skeletal changes induced by early functional treatment of Class III malocclusion: a superimposition study. Am J Orthod Dentofacial Orthop. 1995 Nov;108(5):525-32. 